\title{
Self-Energy Correction to the Two-Photon Decay Width in Hydrogenlike Atoms
}

\author{
Ulrich D. Jentschura ${ }^{1,2}$ \\ ${ }^{I}$ Theoretische Quantendynamik, Physikalisches Institut der Universität Freiburg, \\ Hermann-Herder-Straße 3, 79104 Freiburg im Breisgau, Germany \\ ${ }^{2}$ National Institute of Standards and Technology, Gaithersburg, Maryland 20899-8401
}

\begin{abstract}
We investigate the gauge invariance of the leading logarithmic radiative correction to the two-photon decay width in hydrogenlike atoms. It is shown that an effective treatment of the correction using a Lamb-shift "potential" leads to equivalent results in both the length as well as the velocity gauges provided all relevant correction terms are taken into account. Specifically, the relevant radiative corrections are related to the energies that enter into the propagator denominators, to the Hamiltonian, to the wave functions, and to the energy conservation condition that holds between the two photons; the form of all of these effects is different in the two gauges, but the final result is shown to be gauge invariant, as it should be. Although the actual calculation only involves integrations over nonrelativistic hydrogenic Green functions, the derivation of the leading logarithmic correction can be regarded as slightly more complex than that of other typical logarithmic terms. The dominant radiative correction to the $2 S$ two-photon decay width is found to be $-2.020536(\alpha / \pi)(Z \alpha)^{2} \ln \left[(Z \alpha)^{-2}\right]$ in units of the leading nonrelativistic expression. This result is in agreement with a length-gauge calculation [S. G. Karshenboim and V. G. Ivanov, e-print physics/9702027|, where the coefficient was given as -2.025(1).

PACS numbers: 12.20.Ds, 31.30.Jv, 06.20.Jr, 31.15.-p
\end{abstract}

\section{INTRODUCTION}

The two-photon decay of the metastable $2 S$ level in atomic hydrogen and hydrogenlike systems is a rather intriguing physical phenomenon; it was first investigated by $\mathrm{M}$. Göppert-Mayer a long time ago [1, 2]. The transition involving two quanta limits the lifetime of the metastable $2 S$ resonance, at least for low and medium nuclear charge numbers $Z$. By contrast, the highly suppressed magnetic dipole transition to the $1 S$ ground state has a negligible influence on the decay width [3]. In this article, we evaluate the dominant self-energy radiative correction to the two-photon process. We recall here the known leading-order result [4, 5, 6, 7]

$$
\tau^{-1} \approx \Gamma_{0}=8.229 Z^{6} \mathrm{~s}^{-1}=1.310 Z^{6} \mathrm{~Hz} .
$$

For ionized helium $(Z=2)$, rather accurate experimental verifications of this result exist [8, 9, 10]. Due to its metastability, the $2 \mathrm{~S}$ level in hydrogenlike systems is one of the most accurately defined resonances found in nature. Indeed, it is this very property - the small natural linewidth—which has made possible the high-resolution two-photon spectroscopy of the $1 S-2 S$ transition [11, 12, 13, 14, 15, 16].

The fully relativistic quantum electrodynamic formalism is intricate when applied to bound-state problems [17, 18, 19, 20], but it is often possible to gain a rather good understanding of QED radiative corrections to a particular process if one uses a simplified, NRQED Lagrangian that contains effective operators which then lead to the perturbations that have to be evaluated (see e.g. [21, 22]). Of course, the main difficulty of any bound-state calculation, which is the separation of the two energy scales (scale of binding energy and the energy/mass scale of the free particles), persists in the effective approach. It is necessary to also specify cutoff prescriptions; the artificially introduced scale-separation parameters then cancel at the end of the calculation [21, 22, 23, 24]. Elucidating discussion of the latter point can be found in [25, Ch. 123] and in [26, Ch. 11.4 on p. 493].
Within nonrelativistic quantum electrodynamics (also referred to as NRQED, see [19, 20]), one has the choice between two different forms of the interaction Hamiltonian: the "length" (Yennie) and the "velocity" (Coulomb) gauges. There are certain intriguing issues involved with the gauge invariance in the dynamical nonrelativistic atom-light interaction. Indeed, in order to prove gauge invariance for dynamical processes, it is in many cases necessary to carefully consider the gauge transformation of the atomic wave function in addition to the transformation of the fields. Otherwise, nongauge invariant results are obtained off resonance [27, 28, 29]. In the current situation of radiative corrections to the twophoton decay width, we will show that it is possible to ignore the transformation of the wave function: the two-photon decay width, including the radiative corrections, is invariant under a "hybrid" gauge transformation [28] which involves only the fields, but ignores the gauge transformation of the wave function. In general, the choice of the gauge and the interpretation of physical operators have to be considered very carefully in time-dependent problems (see [30, p. 268] and Refs. [27, 28, 29]).

The gauge invariance of the two-photon decay rate and of the radiative corrections to this effect can be regarded as slightly problematic, partly because the integration over the photon energy is restricted to a finite interval. By contrast, the gauge invariance of the low-energy part of the one-loop selfenergy shift, in an effective NRQED treatment, holds only because one may drop terms whose divergence, for large photon frequency, is stronger than logarithmic [23, see Eq. (3.4) ff.]; in this case gauge invariance would be violated over finite intervals of the virtual photon frequency. It has been one of the main motivations for the current paper to study related questions.

This article is organized as follows: In Sec. II the leading nonrelativistic contribution to the two-photon decay rate is discussed, together with its relation to the NRQED twophoton self-energy. In Sec. IIII the leading logarithmic radia- 
tive correction to the two-photon decay rate is formulated, the discussion is based on a perturbation with an effective potential. Explicit expressions are derived in Secs. IV and $\mathrm{V}$ for the length and velocity gauges, respectively. Gauge invariance is proven in Sec. VI Numerical results are presented in Sec. VIII Conclusions are drawn in Sec. VIII All derivations are presented in some detail, for the sake of transparency.

\section{LEADING-ORDER TWO-PHOTON DECAY RATE}

The decay width of a bound system may be understood naturally as the imaginary part of the self energy [31]. Indeed, the (negative) imaginary part of the self-energy is just $\Gamma / 2$, where $\Gamma$ is the decay width. We discuss the derivation of the two-photon width based on this concept, within nonrelativistic quantum electrodynamics [32].

The formulation of the two-loop self-energy problem within the context of nonrelativistic quantum electrodynamics (NRQED) has been discussed in [33]. We denote by $p^{j}$ the Cartesian components of the momentum operator $\boldsymbol{p}=$ $-\mathrm{i} \nabla$. The expression for the two-loop self-energy shift reads [33, 34]

$$
\begin{aligned}
\Delta & E_{\mathrm{NRQED}}=-\left(\frac{2 \alpha}{3 \pi m^{2}}\right)^{2} \int_{0}^{\epsilon_{1}} d \omega_{1} \omega_{1} \int_{0}^{\epsilon_{2}} d \omega_{2} \omega_{2}\left\{\left\langle p^{i} \frac{1}{H-E+\omega_{1}} p^{j} \frac{1}{H-E+\omega_{1}+\omega_{2}} p^{i} \frac{1}{H-E+\omega_{2}} p^{j}\right\rangle\right. \\
& +\frac{1}{2}\left\langle p^{i} \frac{1}{H-E+\omega_{1}} p^{j} \frac{1}{H-E+\omega_{1}+\omega_{2}} p^{j} \frac{1}{H-E+\omega_{1}} p^{i}\right\rangle \\
& +\frac{1}{2}\left\langle p^{i} \frac{1}{H-E+\omega_{2}} p^{j} \frac{1}{H-E+\omega_{1}+\omega_{2}} p^{j} \frac{1}{H-E+\omega_{2}} p^{i}\right\rangle \\
& +\left\langle p^{i} \frac{1}{H-E+\omega_{1}} p^{i}\left(\frac{1}{H-E}\right)^{\prime} p^{j} \frac{1}{H-E+\omega_{2}} p^{i}\right\rangle \\
& -\frac{1}{2}\left\langle p^{i} \frac{1}{H-E+\omega_{1}} p^{i}\right\rangle\left\langle p^{j}\left(\frac{1}{H-E+\omega_{2}}\right)^{2} p^{i}\right\rangle-\frac{1}{2}\left\langle p^{i} \frac{1}{H-E+\omega_{2}} p^{i}\right\rangle\left\langle p^{j}\left(\frac{1}{H-E+\omega_{1}}\right)^{2} p^{i}\right\rangle \\
& \left.-m\left\langle p^{i} \frac{1}{H-E+\omega_{1}} \frac{1}{H-E+\omega_{2}} p^{i}\right\rangle-\frac{m}{\omega_{1}+\omega_{2}}\left\langle p^{i} \frac{1}{H-E+\omega_{2}} p^{i}\right\rangle-\frac{m}{\omega_{1}+\omega_{2}}\left\langle p^{i} \frac{1}{H-E+\omega_{1}} p^{i}\right\rangle\right\} .
\end{aligned}
$$

All of the matrix elements are evaluated on the reference state $|\phi\rangle$, for which the nonrelativistic Schrödinger wave function is employed. The expression for the two-photon decay width [Eq. (4) below] now follows in a natural way as the imaginary part generated by the sum of the first three terms in curly brackets in Eq. (2). Specifically, the poles are generated upon $\omega_{2}$-integration by the propagator

$$
\frac{1}{H-E+\omega_{1}+\omega_{2}}=\sum_{\phi^{\prime}} \frac{\left|\phi^{\prime}\right\rangle\left\langle\phi^{\prime}\right|}{E^{\prime}-E+\omega_{1}+\omega_{2}}
$$

at $\omega_{2}=E-E^{\prime}-\omega_{1}$. Alternatively, this condition may be expressed as $E-E^{\prime}=\omega_{1}+\omega_{2}$, and represents the energy conservation condition for the two-photon decay. The imaginary part generated by the first three terms in curly brackets of the energy shift (2) is thus seen to yield the two-photon decay width [35].

In view of the above discussion, and in agreement with Shapiro and Breit [4, Eq. (3)], the nonrelativistic expression for the two-photon decay width $\Gamma_{0}$ in the case $|\phi\rangle=|2 S\rangle$ and $\left|\phi^{\prime}\right\rangle=|1 S\rangle$ reads

$$
\begin{aligned}
\Gamma_{0}= & \frac{4}{27} \frac{\alpha^{2}}{\pi} \int_{0}^{\omega_{\max }} d \omega_{1} \omega_{1}^{3} \omega_{2}^{3} \\
& \mid\left\langle\phi^{\prime}\left|x^{i} \frac{1}{H-E+\omega_{2}} x^{i}\right| \phi\right\rangle \\
& +\left.\left\langle\phi^{\prime}\left|x^{i} \frac{1}{H-E+\omega_{1}} x^{i}\right| \phi\right\rangle\right|^{2},
\end{aligned}
$$

where $\omega_{2}=\omega_{\max }-\omega_{1}$ and $\omega_{\max }=E-E^{\prime}$ is the maximum energy that any of the two photons may have. When comparing this expression to Eq. (2) of [36], it should be noted that the quantity $y \mathrm{ibid}$. represents a scaled photon energy. The Einstein summation convention is used throughout this arti- 
cle. Note the following identity [27, 37]

$$
\begin{aligned}
\left\langle\phi^{\prime}\right. & \frac{p^{i}}{m} \frac{1}{H-E+\omega_{1}} \frac{p^{i}}{m}|\phi\rangle \\
& +\left\langle\phi^{\prime}\left|\frac{p^{i}}{m} \frac{1}{H-E+\omega_{2}} \frac{p^{i}}{m}\right| \phi\right\rangle \\
= & -\omega_{1} \omega_{2} m^{2}\left\{\left\langle\phi^{\prime}\left|x^{i} \frac{1}{H-E+\omega_{1}} x^{i}\right| \phi\right\rangle\right. \\
& \left.+\left\langle\phi^{\prime}\left|x^{i} \frac{1}{H-E+\omega_{2}} x^{i}\right| \phi\right\rangle\right\},
\end{aligned}
$$

which is valid at exact resonance $\omega_{1}+\omega_{2}=E-E^{\prime}$. This identity permits a reformulation of the problem in the velocitygauge as opposed to the length-gauge form.

\section{RADIATIVE CORRECTIONS}

We consider a hydrogenlike atom and employ natural units with $\hbar=\epsilon_{0}=c=1$. In order to analyze the radiative correction to the two-photon decay width, one could write down all Feynman diagrams which contribute to the process, and start evaluating them. However, a much more economical understanding into the problem can be gained by considering an approach inspired by effective field theory, or nonrelativistic quantum electrodynamics [19, 20], in which the leading effect due to radiative photons is described by an effective Lambshift potential [38, 39]

$$
\delta V_{\text {Lamb }}=\frac{4}{3} \alpha(Z \alpha) \ln \left[(Z \alpha)^{-2}\right] \frac{\delta^{(3)}(\boldsymbol{r})}{m^{2}} .
$$

In this work we will consider a "standard normalized perturbative local potential" [40]

$$
\delta V=\frac{\pi(Z \alpha)}{m^{2}} \delta^{(3)}(\boldsymbol{r}) .
$$

which is related to $\delta V_{\text {Lamb }}$ by a simple prefactor,

$$
\delta V_{\text {Lamb }}=\frac{4}{3} \frac{\alpha}{\pi} \ln \left[(Z \alpha)^{-2}\right] \delta V .
$$

The corrections to the Hamiltonian, to the energy and to the wavefunction, incurred by the perturbative potential (7), read as follows,

$$
\begin{aligned}
E & \rightarrow E+\delta E, \\
\delta E & =\langle\phi|\delta V| \phi\rangle, \\
H & \rightarrow H+\delta V, \\
|\phi\rangle & \rightarrow|\phi\rangle+|\delta \phi\rangle, \\
|\delta \phi\rangle & =\left(\frac{1}{E-H}\right)^{\prime} \delta V|\phi\rangle .
\end{aligned}
$$

The standard potential (7) leads to a "normalized" energy shift with unit prefactors,

$$
\delta E(n S)=\frac{(Z \alpha)^{4} m}{n^{3}} .
$$

\section{LENGTH GAUGE}

According to (4), the two-photon decay rate $\Gamma_{0}$ of the metastable $2 S$ state is given by

$$
\frac{\Gamma_{0}}{A}=\int_{0}^{\omega_{\max }} d \omega_{1} \omega_{1}^{3} \omega_{2}^{3} \zeta^{2}
$$

where we use the definition

$$
A=\frac{4}{27} \frac{\alpha^{2}}{\pi},
$$

as well as $\omega_{2} \equiv E_{2 S}-E_{1 S}-\omega_{1}$ and $\omega_{\max } \equiv E_{2 S}-E_{1 S}$. The quantity $\zeta$ is given by

$$
\zeta=\zeta_{1}+\zeta_{2},
$$

where

$$
\begin{aligned}
& \zeta_{1}=\left\langle 1 S\left|x^{i} \frac{1}{H-E_{2 S}+\omega_{1}} x^{i}\right| 2 S\right\rangle, \\
& \zeta_{2}=\left\langle 1 S\left|x^{i} \frac{1}{H-E_{1 S}-\omega_{1}} x^{i}\right| 2 S\right\rangle .
\end{aligned}
$$

The perturbation (9) leads to the following replacements, which include the first-order corrections to the various quantities that are relevant to the $2 S$ decay width,

$$
\begin{aligned}
E_{1 S} & \rightarrow E_{1 S}+\delta E_{1 S}, \quad \delta E_{1 S}=\langle 1 S|\delta V| 1 S\rangle, \\
E_{2 S} & \rightarrow E_{2 S}+\delta E_{2 S}, \quad \delta E_{2 S}=\langle 2 S|\delta V| 2 S\rangle, \\
|1 S\rangle & \rightarrow|1 S\rangle+\left(\frac{1}{E_{1 S}-H}\right)^{\prime} \delta V|1 S\rangle, \\
|2 S\rangle & \rightarrow|2 S\rangle+\left(\frac{1}{E_{2 S}-H}\right)^{\prime} \delta V|2 S\rangle, \\
\omega_{2} & \rightarrow \omega_{2}+\delta \omega_{2}, \quad \delta \omega_{2}=\delta E_{2 S}-\delta E_{1 S} .
\end{aligned}
$$

The latter correction ensures that a perturbed energy conservation condition is fulfilled,

$$
\omega_{1}+\omega_{2}+\delta \omega_{2}=E_{2 S}-E_{1 S}+\left(\delta E_{2 S}-\delta E_{1 S}\right),
$$

i.e. that the two photon frequencies add up to the perturbed transition frequency.

The first-order self-energy correction $\delta \Gamma$ to the two-photon decay rate may be expressed as

$$
\frac{\delta \Gamma}{B}=2 \int_{0}^{\omega_{\max }} d \omega_{1} \omega_{1}^{3} \omega_{2}^{3} \zeta \delta \zeta+3 \delta \omega_{2} \int_{0}^{\omega_{\max }} d \omega_{1} \omega_{1}^{3} \omega_{2}^{2} \zeta^{2},
$$

where the correction $\delta \zeta$ is the sum of six terms,

$$
\delta \zeta=\sum_{j=1}^{6} \delta \zeta_{j}
$$


to be defined as follows, and the second term on the right-hand side of (16) is due to perturbed energy conservation condition. The quantity $B$ may be inferred from (4), (7) and (8) as

$$
B=\frac{16}{81} \frac{\alpha^{3}}{\pi^{2}} \ln \left[(Z \alpha)^{-2}\right] .
$$

The terms $\delta \zeta_{1}$ and $\delta \zeta_{2}$ are related to energy perturbations to the matrix elements,

$$
\begin{aligned}
& \delta \zeta_{1}=\left\langle 1 S\left|x^{i}\left(\frac{1}{H-E_{2 S}+\omega_{1}}\right)^{2} x^{i}\right| 2 S\right\rangle\langle 2 S|\delta V| 2 S\rangle, \\
& \delta \zeta_{2}=\langle 1 S|\delta V| 1 S\rangle\left\langle 1 S\left|x^{i}\left(\frac{1}{H-E_{1 S}-\omega_{1}}\right)^{2} x^{i}\right| 2 S\right\rangle,
\end{aligned}
$$

whereas the terms $\delta \zeta_{3,4,5,6}$ are perturbations to the initial- and final-state wave functions,

$$
\begin{aligned}
\delta \zeta_{3} & =\left\langle 1 S\left|x^{i} \frac{1}{H-E_{2 S}+\omega_{1}} x^{i}\left(\frac{1}{E_{2 S}-H}\right)^{\prime} \delta V\right| 2 S\right\rangle, \\
\delta \zeta_{4} & =\left\langle 1 S\left|x^{i} \frac{1}{H-E_{1 S}-\omega_{1}} x^{i}\left(\frac{1}{E_{2 S}-H}\right)^{\prime} \delta V\right| 2 S\right\rangle, \\
\delta \zeta_{5} & =\left\langle 1 S\left|\delta V\left(\frac{1}{E_{1 S}-H}\right)^{\prime} x^{i} \frac{1}{H-E_{2 S}+\omega_{1}} x^{i}\right| 2 S\right\rangle, \\
\delta \zeta_{6} & =\left\langle 1 S\left|\delta V\left(\frac{1}{E_{1 S}-H}\right)^{\prime} x^{i} \frac{1}{H-E_{1 S}-\omega_{1}} x^{i}\right| 2 S\right\rangle .
\end{aligned}
$$

\section{VELOCITY GAUGE}

We now discuss the evaluation of radiative corrections in the velocity gauge, where the interaction Hamiltonian is given by

$$
H_{\mathrm{int}}^{\prime}=-e \frac{\boldsymbol{p} \cdot \boldsymbol{A}}{m}+e^{2} \frac{\boldsymbol{A}^{2}}{2 m^{2}} .
$$

According to (4) and (5), the leading-order decay rate in the velocity gauge is

$$
\frac{\Gamma_{0}^{\prime}}{A}=\int_{0}^{\omega_{\max }} d \omega_{1} \omega_{1} \omega_{2} \xi^{2}
$$

where $A$ is defined in (12), $\omega_{2} \equiv E_{2 S}-E_{1 S}-\omega_{1}$ and $\omega_{\max } \equiv$ $E_{2 S}-E_{1 S}$. The quantity $\xi$ is the sum of two terms,

$$
\xi=\xi_{1}+\xi_{2}
$$

where

$$
\begin{aligned}
& \xi_{1}=\left\langle 1 S\left|\frac{p^{i}}{m} \frac{1}{H-E_{2 S}+\omega_{1}} \frac{p^{i}}{m}\right| 2 S\right\rangle, \\
& \xi_{2}=\left\langle 1 S\left|\frac{p^{i}}{m} \frac{1}{H-E_{1 S}-\omega_{1}} \frac{p^{i}}{m}\right| 2 S\right\rangle .
\end{aligned}
$$

Gauge invariance of the leading-order decay-rate [see Eqs. (11) and (21)

$$
\Gamma_{0}=\Gamma_{0}^{\prime}
$$

immediately follows from Eq. (5); this equation may be rewritten in a compact form as

$$
\xi=-\omega_{1} \omega_{2} \zeta
$$

Equation 24 may be proven easily by repeated application of the commutator relation(s)

$$
\frac{p^{i}}{m}=\mathrm{i}\left[H-E_{2 S}+\omega_{1}, x^{i}\right]=\mathrm{i}\left[H-E_{1 S}-\omega_{1}, x^{i}\right] .
$$

Now the first-order correction to the two-photon decay rate, in the velocity gauge, is

$$
\frac{\delta \Gamma^{\prime}}{B}=2 \int_{0}^{\omega_{\max }} d \omega_{1} \omega_{1} \omega_{2} \xi \delta \xi+\delta \omega_{2} \int_{0}^{\omega_{\max }} d \omega_{1} \omega_{1} \xi^{2},
$$

where the prime denotes the velocity-gauge form of the correction and $B$ is defined in (18). We desire to show that $\delta \Gamma=\delta \Gamma^{\prime}$. 
The correction $\delta \xi$ finds a natural representation as the sum of eight terms,

$$
\delta \xi=\sum_{j=1}^{8} \delta \xi_{j}
$$

In analogy to $19 \mathrm{a}$ and $19 \mathrm{~b}, \delta \xi_{1}$ and $\delta \xi_{2}$ are energy perturbations,

$$
\begin{aligned}
\delta \xi_{1} & =\left\langle 1 S\left|\frac{p^{i}}{m}\left(\frac{1}{H-E_{2 S}+\omega_{1}}\right)^{2} \frac{p^{i}}{m}\right| 2 S\right\rangle\langle 2 S|\delta V| 2 S\rangle, \\
\delta \xi_{2} & =\langle 1 S|\delta V| 1 S\rangle\left\langle 1 S\left|\frac{p^{i}}{m}\left(\frac{1}{H-E_{1 S}-\omega_{1}}\right)^{2} \frac{p^{i}}{m}\right| 2 S\right\rangle .
\end{aligned}
$$

The terms $\delta \xi_{3,4,5,6}$ are perturbations to the initial- and final-state wave functions,

$$
\begin{aligned}
\delta \xi_{3} & =\left\langle 1 S\left|\frac{p^{i}}{m} \frac{1}{H-E_{2 S}+\omega_{1}} \frac{p^{i}}{m}\left(\frac{1}{E_{2 S}-H}\right)^{\prime} \delta V\right| 2 S\right\rangle, \\
\delta \xi_{4} & =\left\langle 1 S\left|\frac{p^{i}}{m} \frac{1}{H-E_{1 S}-\omega_{1}} \frac{p^{i}}{m}\left(\frac{1}{E_{2 S}-H}\right)^{\prime} \delta V\right| 2 S\right\rangle, \\
\delta \xi_{5} & =\left\langle 1 S\left|\delta V\left(\frac{1}{E_{1 S}-H}\right)^{\prime} \frac{p^{i}}{m} \frac{1}{H-E_{2 S}+\omega_{1}} \frac{p^{i}}{m}\right| 2 S\right\rangle, \\
\delta \xi_{6} & =\left\langle 1 S\left|\delta V\left(\frac{1}{E_{1 S}-H}\right)^{\prime} \frac{p^{i}}{m} \frac{1}{H-E_{1 S}-\omega_{1}} \frac{p^{i}}{m}\right| 2 S\right\rangle .
\end{aligned}
$$

Finally, $\delta \xi_{7,8}$ are due to the seagull term,

$$
\begin{aligned}
& \delta \xi_{7}=-\frac{3}{m}\left\langle 1 S\left|\left(\frac{1}{E_{2 S}-H}\right)^{\prime} \delta V\right| 2 S\right\rangle, \\
& \delta \xi_{8}=-\frac{3}{m}\left\langle 1 S\left|\delta V\left(\frac{1}{E_{1 S}-H}\right)^{\prime}\right| 2 S\right\rangle .
\end{aligned}
$$

\section{PROOF OF GAUGE INVARIANCE}

Here, we merely present the results of the analysis carried out in detail in App. @ Indeed, using Eqs. A1a — A1f , as well as $\mathrm{A2 \textrm {a }}$ and $\mathrm{A} 2 \mathrm{~b}$, we obtain the compact relation

$$
\delta \xi=-\omega_{1} \omega_{2} \delta \zeta-\delta \omega_{2} \omega_{1} \zeta
$$


In view of this relation, we can rewrite (16) and (26) using (29),

$$
\begin{aligned}
\frac{\delta \Gamma^{\prime}}{B} & =2 \int_{0}^{\omega_{\max }} d \omega_{1} \omega_{1} \omega_{2} \xi \delta \xi+\delta \omega_{2} \int_{0}^{\omega_{\max }} d \omega_{1} \omega_{1} \xi^{2} \\
& =2 \int_{0}^{\omega_{\max }} d \omega_{1} \omega_{1} \omega_{2}\left(-\omega_{1} \omega_{2} \zeta\right)\left[-\omega_{1} \omega_{2} \delta \zeta-\delta \omega_{2} \omega_{1} \zeta\right]+\delta \omega_{2} \int_{0}^{\omega_{\max }} d \omega_{1} \omega_{1}^{3} \omega_{1}^{2} \zeta^{2} \\
& =2 \int_{0}^{\omega_{\max }} d \omega_{1} \omega_{1}^{3} \omega_{2}^{3} \zeta \delta \zeta+(2+1) \delta \omega_{2} \int_{0}^{\omega_{\max }} d \omega_{1} \omega_{1}^{3} \omega_{1}^{2} \zeta^{2} \\
& =\frac{\delta \Gamma}{B} .
\end{aligned}
$$

This proves the gauge invariance $\delta \Gamma=\delta \Gamma^{\prime}$ of the logarithmic radiative corrections to the two-photon decay rate of the metastable $2 S$ state in hydrogenlike systems. The gauge invariance of the leading-order decay rate $\left(\Gamma_{0}=\Gamma_{0}^{\prime}\right)$ has been indicated in Eq. (23).

\section{NUMERICAL RESULTS}

(Leading order.) We recall that, according to (4), the wellknown leading-order nonrelativistic effect $\Gamma_{0}$ is of the order of $\alpha^{2}(Z \alpha)^{6}$. The result for the two-photon decay width of the metastable $2 S$ state is

$$
\Gamma_{0}=0.001318222 \alpha^{2}(Z \alpha)^{6} m .
$$

This translates into

$$
\begin{aligned}
\Gamma_{0} & =8.229351997 Z^{6} \mathrm{~s}^{-1} \\
& =1.309742049 Z^{6} \mathrm{~Hz}
\end{aligned}
$$

(Radiative correction.) In view of Eqs. (7) and (8), the leading logarithmic radiative correction $\delta \Gamma$ is of the order of

$$
\delta \Gamma \sim \alpha^{3}(Z \alpha)^{8} \ln \left[(Z \alpha)^{-2}\right] m
$$

i.e. of relative order $\alpha(Z \alpha)^{2} \ln \left[(Z \alpha)^{-2}\right]$ with respect to $\Gamma_{0}$. In the length gauge, the relevant expression for $\delta \Gamma$ can be found in Eq. 116. [For clarity, we would like to indicate that the correction $\delta \omega_{2}$ occurring in the expression (16) is defined in (14), the quantity $\zeta$ can be found in (13), and the terms $\delta \zeta_{i}$ $(i=1, \ldots, 6)$ are defined in Eq. (19).] In the velocity gauge, the relevant expression for $\delta \Gamma^{\prime}$ can be found in (26), with the $\delta \xi_{i}(i=1, \ldots, 8)$ being defined in Eq. (28).

According to (16) and (26), both $\delta \Gamma$ as well as $\delta \Gamma^{\prime}$ find a natural representation as the sum of two terms, the first of which summarizes the perturbations to the matrix elements, and the second is a consequence of the perturbed energy conservation condition for the transition. Gauge invariance $\delta \Gamma=\delta \Gamma^{\prime}$ has been shown in Sec.VI yet it is instructive to observe that there are indeed considerable cancellations among the two contributions to $\delta \Gamma$ and $\delta \Gamma^{\prime}$. Specifically, we have from the first and the second terms on the right-hand sides of
(16) and (26), respectively,

$$
\begin{aligned}
\frac{\delta \Gamma}{\Gamma_{0}} & =(29.542-31.562) \frac{\alpha}{\pi}(Z \alpha)^{2} \ln \left[(Z \alpha)^{-2}\right], \\
\frac{\delta \Gamma^{\prime}}{\Gamma_{0}^{\prime}} & =(8.500-10.521) \frac{\alpha}{\pi}(Z \alpha)^{2} \ln \left[(Z \alpha)^{-2}\right] .
\end{aligned}
$$

[The cancellations appear to be typical for radiative corrections to decay rates; this has recently been observed in connection with radiative corrections to the one-photon decay of $P$ states [41].]

The final result for the leading logarithmic correction to the decay width of the metastable $2 S$ state is

$$
\frac{\delta \Gamma}{\Gamma_{0}}=\frac{\delta \Gamma^{\prime}}{\Gamma_{0}}=-2.020536 \frac{\alpha}{\pi}(Z \alpha)^{2} \ln \left[(Z \alpha)^{-2}\right] .
$$

The calculation of $\delta \zeta$ and $\delta \xi$ involves expressions analogous to those encountered in [40]. In [36], the coefficient has been given as $-2.025(1)$, which is in agreement with the current calculation. [There is a misprint in the overall sign of the correction as given in the abstract of [36]; one should follow the sign indicated in Eq. (8) ibid.]

The result (36), converted to Hertz and/or inverse seconds, reads

$$
\begin{aligned}
\delta \Gamma & =-3.273 \times 10^{-7} Z^{8} \ln \left[137^{2} Z^{-2}\right] \mathrm{Hz} \\
& =-2.057 \times 10^{-6} Z^{8} \ln \left[137^{2} Z^{-2}\right] \mathrm{s}^{-1} .
\end{aligned}
$$

For low $Z$, the highly suppressed $M 1$ one-photon decay $2 S \rightarrow 1 S$ is numerically smaller than the radiative correction 37] to the two-photon decay (see Refs. [42, 43, 44, 45, 46]) because it lacks the large logarithm:

$$
\Gamma_{M 1}=2.496 \times 10^{-6} Z^{10} \mathrm{~s}^{-1} .
$$

All results indicated in this article for $\Gamma$ and $\delta \Gamma$ relate to the metastable $2 S$ state; however the approach may easily be generalized to the two-photon decay of other states. 


\section{CONCLUSIONS}

In the current investigation, the derivation of the leading radiative correction to the two-photon decay width of the metastable $2 S$ state in hydrogenlike atoms has been based on the effective "radiative potential" (6) discussed in Sec. III It has been shown that the gauge invariance of the corrections holds due to the interplay of corrections to the transition matrix elements on the one hand and corrections due to perturbed energy conservation conditions on the other hand [first and second terms on the right-hand sides of (16) and 26), respectively]. The corrections to the transition matrix elements are again divided into corrections to the wave function (these were referred to as the $d$-terms in [36]), and to the energies that enter into the propagator denominators, which were termed $f$ in the length-gauge calculation [36]. The length- and velocitygauge forms of the correction are discussed in Secs. [V] and V The gauge invariance of the radiative correction holds (even) on the level of the effective treatment as implied by the radiative potential (6), as shown in Sec. DI] All derivations are presented in some detail, for clarity and transparency. The numerical evaluation in Sec.VII follows immediately.

There are two more results of the current paper, probably of rather minor importance, which should only briefly be mentioned: first of all, the relativistic result (without radiative corrections) for the decay rate at $Z=1$ has previously been indicated as $8.229 \mathrm{~s}^{-1}[47,48,49,50]$, whereas in [4], the (nonrelativistic) result has been indicated as $8.226 \pm 0.001$ inverse seconds. The current investigation [Eq. (32)] confirms that the discrepancy has been due to a certain overestimation of the numerical accuracy in the early nonrelativistic calculation [4], not due to a conceivable large relativistic shift. Second, the discussion in Sec. II clarifies that the concept of a decay width as an imaginary part of a self-energy [31] generalizes to the two-loop self-energy shift, in which case the imaginary part gives rise to the two-photon decay width.

The leading-order nonrelativistic contribution to the twophoton decay width is of the order of $\alpha^{2}(Z \alpha)^{6} m c^{2}$ (see Sec. (II). The self-energy radiative correction to the twophoton decay is of the order of $\alpha^{3}(Z \alpha)^{8} \ln \left[(Z \alpha)^{-2}\right] m c^{2}$, as discussed in Sec. VII with explicit results indicated in
Eqs. (36) and (37). It would be interesting to evaluate also the constant term of relative order $\alpha(Z \alpha)^{2}$. This term supplements the logarithm evaluated here which is of relative order $\alpha(Z \alpha)^{2} \ln \left[(Z \alpha)^{-2}\right]$. According to our experience, in boundstate calculations, the nonlogarithmic, constant term has an opposite sign as compared to the leading logarithm, and its magnitude is two or three times larger than the coefficient of the logarithm. This is true for radiative corrections [41] as well as Lamb-shift effects [51, 52].

One should note a rather general interest in various intriguing details related to the two-photon decay process, which are not restricted to the search for conceivable parity admixtures to the $2 S$ state (see e.g. [53, 54]). Although accurate measurements of integrated decay rates are difficult [55], there is some hope that in low- $Z$ and middle- $Z$ ionic systems, experiments will eventually profit from the possibilities offered by electron-beam ion traps, especially when combined with conceivable x-ray lasers that could be used in order to excite the trapped ions into the metastable states.

Finally, we recall that accurate measurements of the twophoton decay width test the $2 S$ state for parity-violating $2 P$ admixtures and can therefore be used as a test for a conceivable electron or nuclear (electric) dipole moment or for interactions via "anapole" or "pseudocharge" currents [56, 57, 58]. One particularly interesting investigation on hydrogenlike $\mathrm{Ar}^{17+}$, with an elucidating discussion of the issues related to parity admixtures, has been given in [59].

\section{Acknowledgments}

The author acknowledges elucidating discussions with Holger Gies and Wilhelm Becker on questions related to the gauge invariance, and insightful conversations with Krzysztof Pachucki regarding quantum electrodynamic effects in bound systems. The author wishes to thank Gordon Drake for very helpful remarks. Sabine Jentschura is acknowledged for carefully reading the manuscript. The stimulating atmosphere at the National Institute of Standards and Technology has contributed to the completion of this project.

\section{APPENDIX A: RELATIONS AMONG MATRIX ELEMENTS}

In this appendix, we present in detail the relations needed for the proof of the identity (29). For $\delta \xi_{1}$ as defined in (28a), we have

$$
\begin{aligned}
& \left\langle 1 S\left|\frac{p^{i}}{m}\left(\frac{1}{H-E_{2 S}+\omega_{1}}\right)^{2} \frac{p^{i}}{m}\right| 2 S\right\rangle=-\omega_{1} \omega_{2}\left\langle 1 S\left|x^{i}\left(\frac{1}{H-E_{2 S}+\omega_{1}}\right)^{2} x^{i}\right| 2 S\right\rangle \\
& \quad+\left(\omega_{2}-\omega_{1}\right)\left\langle 1 S\left|x^{i} \frac{1}{H-E_{1 S}+\omega_{1}} x^{i}\right| 2 S\right\rangle+\left\langle 1 S\left|x^{i} x^{i}\right| 2 S\right\rangle .
\end{aligned}
$$


We notice the term $\delta \zeta_{1}$ emerge on the right-hand side [see Eq. [19a] ]. The corresponding relation for $\delta \xi_{2}$ reads

$$
\begin{aligned}
& \left\langle 1 S\left|\frac{p^{i}}{m}\left(\frac{1}{H-E_{1 S}-\omega_{1}}\right)^{2} \frac{p^{i}}{m}\right| 2 S\right\rangle=-\omega_{1} \omega_{2}\left\langle 1 S\left|x^{i}\left(\frac{1}{H-E_{1 S}-\omega_{1}}\right)^{2} x^{i}\right| 2 S\right\rangle \\
& \quad+\left(\omega_{1}-\omega_{2}\right)\left\langle 1 S\left|x^{i} \frac{1}{H-E_{1 S}-\omega_{1}} x^{i}\right| 2 S\right\rangle+\left\langle 1 S\left|x^{i} x^{i}\right| 2 S\right\rangle .
\end{aligned}
$$

For $\delta \xi_{3}$, the following relation is useful,

$$
\begin{aligned}
& \left\langle 1 S\left|\frac{p^{i}}{m} \frac{1}{H-E_{2 S}+\omega_{1}} \frac{p^{i}}{m}\left(\frac{1}{E_{2 S}-H}\right)^{\prime} \delta V\right| 2 S\right\rangle=-\omega_{1} \omega_{2}\left\langle 1 S\left|x^{i} \frac{1}{H-E_{2 S}+\omega_{1}} x^{i}\left(\frac{1}{E_{2 S}-H}\right)^{\prime} \delta V\right| 2 S\right\rangle \\
& -\omega_{2}\left\langle 1 S\left|x^{i} \frac{1}{H-E_{2 S}+\omega_{1}} x^{i}\right| 2 S\right\rangle\langle 2 S|\delta V| 2 S\rangle+\left\langle 1 S\left|x^{i}\left(H-E_{2 S}+\omega_{2}\right) x^{i}\left(\frac{1}{E_{2 S}-H}\right)^{\prime} \delta V\right| 2 S\right\rangle \\
& -\left\langle 1 S\left|x^{i} x^{i}\right| 2 S\right\rangle\langle 2 S|\delta V| 2 S\rangle .
\end{aligned}
$$

For $\delta \xi_{4}$, we have

$$
\begin{aligned}
& \left\langle 1 S\left|\frac{p^{i}}{m} \frac{1}{H-E_{1 S}-\omega_{1}} \frac{p^{i}}{m}\left(\frac{1}{E_{2 S}-H}\right)^{\prime} \delta V\right| 2 S\right\rangle=-\omega_{1} \omega_{2}\left\langle 1 S\left|x^{i} \frac{1}{H-E_{1 S}-\omega_{1}} x^{i}\left(\frac{1}{E_{2 S}-H}\right)^{\prime} \delta V\right| 2 S\right\rangle \\
& \quad-\omega_{1}\left\langle 1 S\left|x^{i} \frac{1}{H-E_{1 S}-\omega_{1}} x^{i}\right| 2 S\right\rangle\langle 2 S|\delta V| 2 S\rangle+\left\langle 1 S\left|x^{i}\left(H-E_{1 S}-\omega_{2}\right) x^{i}\left(\frac{1}{E_{2 S}-H}\right)^{\prime} \delta V\right| 2 S\right\rangle \\
& \quad-\left\langle 1 S\left|x^{i} x^{i}\right| 2 S\right\rangle\langle 2 S|\delta V| 2 S\rangle .
\end{aligned}
$$

The term $\delta \xi_{5}$ may be reformulated according to

$$
\begin{aligned}
& \left\langle 1 S\left|\delta V\left(\frac{1}{E_{1 S}-H}\right)^{\prime} \frac{p^{i}}{m} \frac{1}{H-E_{2 S}+\omega_{1}} \frac{p^{i}}{m}\right| 2 S\right\rangle=-\omega_{1} \omega_{2}\left\langle 1 S\left|\delta V\left(\frac{1}{E_{1 S}-H}\right)^{\prime} x^{i} \frac{1}{H-E_{2 S}+\omega_{1}} x^{i}\right| 2 S\right\rangle \\
& \quad+\omega_{1}\langle 1 S|\delta V| 1 S\rangle\left\langle 1 S\left|x^{i} \frac{1}{H-E_{1 S}-\omega_{1}} x^{i}\right| 2 S\right\rangle+\left\langle 1 S\left|\delta V\left(\frac{1}{E_{1 S}-H}\right)^{\prime} x^{i}\left(H-E_{1 S}-\omega_{1}\right) x^{i}\right| 2 S\right\rangle \\
& \quad-\langle 1 S|\delta V| 1 S\rangle\left\langle 1 S\left|x^{i} x^{i}\right| 2 S\right\rangle .
\end{aligned}
$$

Finally, we have for $\delta \xi_{6}$

$$
\begin{aligned}
& \left\langle 1 S\left|\delta V\left(\frac{1}{E_{1 S}-H}\right)^{\prime} \frac{p^{i}}{m} \frac{1}{H-E_{1 S}-\omega_{1}} \frac{p^{i}}{m}\right| 2 S\right\rangle=-\omega_{1} \omega_{2}\left\langle 1 S\left|\delta V\left(\frac{1}{E_{1 S}-H}\right)^{\prime} x^{i} \frac{1}{H-E_{1 S}-\omega_{1}} x^{i}\right| 2 S\right\rangle \\
& \quad+\omega_{2}\langle 1 S|\delta V| 1 S\rangle\left\langle 1 S\left|x^{i} \frac{1}{H-E_{1 S}-\omega_{1}} x^{i}\right| 2 S\right\rangle+\left\langle 1 S\left|\delta V\left(\frac{1}{E_{1 S}-H}\right)^{\prime} x^{i}\left(H-E_{2 S}+\omega_{1}\right) x^{i}\right| 2 S\right\rangle \\
& \quad-\langle 1 S|\delta V| 1 S\rangle\left\langle 1 S\left|x^{i} x^{i}\right| 2 S\right\rangle .
\end{aligned}
$$


However, the relations A1a - A1f are not yet sufficient in order to proceed with the proof of gauge invariance. We also need

$$
\begin{aligned}
& \left\langle 1 S\left|x^{i}\left[\left(H-E_{1 S}\right)+\left(H-E_{2 S}\right)\right] x^{i}\left(\frac{1}{E_{2 S}-H}\right)^{\prime} \delta V\right| 2 S\right\rangle \\
& =\frac{3}{m}\left\langle 1 S\left|\left(\frac{1}{E_{2 S}-H}\right)^{\prime} \delta V\right| 2 S\right\rangle+\left\langle 1 S\left|x^{i} x^{i}\right| 2 S\right\rangle\langle 2 S|\delta V| 2 S\rangle \\
& \left\langle 1 S\left|\delta V\left(\frac{1}{E_{1 S}-H}\right)^{\prime} x^{i}\left[\left(H-E_{1 S}\right)+\left(H-E_{2 S}\right)\right] x^{i}\right| 2 S\right\rangle \\
& =\frac{3}{m}\left\langle 1 S\left|\delta V\left(\frac{1}{E_{1 S}-H}\right)^{\prime}\right| 2 S\right\rangle+\langle 1 S|\delta V| 1 S\rangle\left\langle 1 S\left|x^{i} x^{i}\right| 2 S\right\rangle
\end{aligned}
$$

We notice the (negative of) the seagull terms $28 \mathrm{~g}$ and $28 \mathrm{~h}$ emerge.

[1] M. Göppert, Naturwissenschaften 17, 932 (1929).

[2] M. Göppert-Mayer, Ann. Phys. (Leipzig) 9, 273 (1931).

[3] R. Marrus and P. J. Mohr, Adv. At. Mol. Phys. 14, 181 (1978).

[4] J. Shapiro and G. Breit, Phys. Rev. 113, 179 (1959).

[5] B. A. Zon and L. P. Rapoport, Pis'ma v. Zh. Éksp. Teor. Fiz. 7, 70 (1968), [JETP Lett. 7, 52 (1968)].

[6] S. Klarsfeld, Phys. Lett. A 30, 382 (1969).

[7] The numerical prefactors of the width is different when expressed in inverse seconds and alternatively in $\mathrm{Hz}$. The following remarks are meant to clarify this situation. In order to obtain the width in $\mathrm{Hz}$, one should interpret the imaginary part of the self-energy [31] as $\Gamma / 2$, and do the same conversion as for the real part of the energy, i.e. divide by $h$, not $\hbar$. This gives the width in $\mathrm{Hz}$. The unit $\mathrm{Hz}$ corresponds to cycles/second. In order to obtain the lifetime in inverse seconds, which is radians per second, one has to multiply the previous result by a factor of $2 \pi$. This result may alternatively be obtained by dividing $\Gamma$, i.e. the imaginary part of the energy, by $\hbar$, not $h$. The general paradigm is that in order to evaluate an energy in units of $\mathrm{Hz}$, one should use the relation $E=h \nu$, whereas for a conversion of an imaginary part of an energy to the inverse lifetime, one should use $\Gamma=\hbar \tau^{-1}$. As calculated in Refs. [4, 6], the width of the metastable $2 S$ state in atomic hydrogenlike systems is $8.229 Z^{6} \mathrm{~s}^{-1}$ (inverse seconds). At $Z=1$, this is equivalent to the "famous" value of $1.3 \mathrm{~Hz}$ which is nowadays most frequently quoted in the literature.

[8] M. H. Prior, Phys. Rev. Lett. 29, 611 (1972).

[9] C. A. Kocher, J. E. Clendenin, and R. Novick, Phys. Rev. Lett. 29, 615 (1972).

[10] E. A. Hinds, J. E. Clendenin, and R. Novick, Phys. Rev. A 17, 670 (1978).

[11] T. Udem, A. Huber, B. Gross, J. Reichert, M. Prevedelli, M. Weitz, and T. W. Hänsch, Phys. Rev. Lett. 79, 2646 (1997).

[12] A. Huber, B. Gross, M. Weitz, and T. W. Hänsch, Phys. Rev. A 59, 1844 (1999).

[13] J. Reichert, M. Niering, R. Holzwarth, M. Weitz, T. Udem, and T. W. Hänsch, Phys. Rev. Lett. 84, 3232 (2000).

[14] M. Niering, R. Holzwarth, J. Reichert, P. Pokasov, T. Udem, M. Weitz, T. W. Hänsch, P. Lemonde, G. Santarelli, M. Abgrall, P.
Laurent, C. Salomon, and A. Clairon, Phys. Rev. Lett. 84, 5496 (2000).

[15] B. de Beauvoir, F. Nez, L. Julien, B. Cagnac, F. Biraben, D. Touahri, L. Hilico, O. Acef, A. Clairon, and J. J. Zondy, Phys. Rev. Lett. 78, 440 (1997).

[16] B. de Beauvoir, C. Schwob, O. Acef, L. Jozefowski, L. Hilico, F. Nez, L. Julien, A. Clairon, and F. Biraben, Eur. Phys. J. D 12, 61 (2000).

[17] S. S. Schweber, An Introduction to Relativistic Quantum Field Theory (Harper \& Row, New York, NY, 1961).

[18] C. Itzykson and J. B. Zuber, Quantum Field Theory (McGrawHill, New York, NY, 1980).

[19] W. E. Caswell and G. P. Lepage, Phys. Lett. B 167, 437 (1986).

[20] B. R. Holstein, Am. J. Phys. 72, 333 (2004).

[21] T. Kinoshita and M. Nio, Phys. Rev. D 53, 4909 (1996).

[22] M. Nio and T. Kinoshita, Phys. Rev. D 55, 7267 (1997).

[23] K. Pachucki, Ann. Phys. (N.Y.) 226, 1 (1993).

[24] U. D. Jentschura and K. Pachucki, Phys. Rev. A 54, 1853 (1996).

[25] V. B. Berestetskii, E. M. Lifshitz, and L. P. Pitaevskii, Quantum Electrodynamics (in Russian, Volume IVb of the Textbook Series of Theoretical Physics by L. D. Landau and E. M. Lifshitz) (PhysMatLit, Moscow, 1989).

[26] S. Weinberg, The Quantum Theory of Fields: Volume 1, Foundations (Cambridge University Press, Cambridge, 1995).

[27] D. H. Kobe, Phys. Rev. Lett. 40, 538 (1978).

[28] R. R. Schlicher, W. Becker, J. Bergou, and M. O. Scully, in Quantum Electrodynamics and Quantum Optics (Plenum, New York, 1984), pp. 405-441.

[29] W. E. Lamb, R. R. Schlicher, and M. O. Scully, Phys. Rev. A 36, 2763 (1987).

[30] W. E. Lamb, Phys. Rev. 85, 259 (1952).

[31] R. Barbieri and J. Sucher, Nucl. Phys. B 134, 155 (1978).

[32] In a number of cases, the formulation of a quantum electrodynamic bound-state problem may be simplified drastically when employing the concepts of an effective low-energy field theory known as nonrelativistic quantum electrodynamics [19]. The basic idea consists in a correspondence between fully relativistic quantum electrodynamics and effective low-energy cou- 
plings between the electron and the radiation field, which may still lead to ultraviolet divergent expressions. However, the ultraviolet divergences may be matched against effective highenergy operators, which leads to a cancellation of the cut-off parameters. Within the context of higher-order binding corrections to the one-loop self-energy problem, this has been discussed in [23, 24].

[33] K. Pachucki, Phys. Rev. A 63, 042503 (2001).

[34] Regarding Eq. 2, we take the opportunity to clarify that the equivalent expression in Eq. (31) of [39] contains two typographical errors: (i) the square of the prefactor $2 \alpha /\left(3 \pi \mathrm{m}^{2}\right)$ should be added, and (ii) the photon energies $\omega_{1}$ and $\omega_{2}$ in the propagator denominators of the second and third term in curly brackets in Eq. (31) of [39] should be interchanged as indicated in Eq. [2]. The further calculations described in [39], especially the double logarithms indicated in Eqs. (32) and (33) of [39], do not receive any corrections.

[35] The other terms in (2) [not only the first three in curly brackets!] may also generate imaginary parts, but they do so only if the reference state is a higher excited $S$ or $P$ state in which case one-photon decay is possible. Indeed, for higher excited $S$ or $P$ states, the additional imaginary energy shifts find a natural interpretation as radiative corrections to the one-photon decay width of these states [41].

[36] S. G. Karshenboim and V. G. Ivanov, Radiative Corrections to the $2 E 1$ Decay Rate of the $2 s$-State in Hydrogen-Like Atoms, e-print physics/9702027 a related publication is S. G. Karshenboim and V. G. Ivanov, Opt. Spectrosc. 83, 1 (1997).

[37] F. Bassani, J. J. Forney, and A. Quattropani, Phys. Rev. Lett. 39, 1070 (1977).

[38] S. G. Karshenboim, J. Phys. B 29, L29 (1996).

[39] U. D. Jentschura and I. Nandori, Phys. Rev. A 66, 022114 (2002).

[40] U. D. Jentschura, J. Phys. A 36, L229 (2003).
[41] J. Sapirstein, K. Pachucki, and K. T. Cheng, Phys. Rev. A 69, 022113 (2004).

[42] G. Breit and E. Teller, Astrophysical Journal 91, 215 (1940).

[43] H. A. Bethe and E. E. Salpeter, Quantum Mechanics of Oneand Two-Electron Atoms (Springer, Berlin, 1957).

[44] G. W. F. Drake, Phys. Rev. A 3, 908 (1971).

[45] G. Feinberg and J. Sucher, Phys. Rev. Lett. 26, 681 (1971).

[46] W. R. Johnson, Phys. Rev. Lett. 29, 1142 (1972).

[47] S. P. Goldman and G. W. F. Drake, Phys. Rev. A 24, 183 (1981).

[48] F. A. Parpia and W. R. Johnson, Phys. Rev. A 26, 1142 (1982).

[49] G. W. F. Drake, Phys. Rev. A 34, 2871 (1986).

[50] A. Derevianko and W. R. Johnson, Phys. Rev. A 56, 1288 (1986).

[51] J. Sapirstein and D. R. Yennie, in Quantum Electrodynamics, Vol. 7 of Advanced Series on Directions in High Energy Physics, edited by T. Kinoshita (World Scientific, Singapore, 1990), pp. 560-672.

[52] The accuracy of the present calculation for the total radiative effects should therefore be estimated as roughly $20 \%$ for $Z=1$ and $Z=2$.

[53] P. J. Mohr, Phys. Rev. Lett. 40, 854 (1978).

[54] A. J. Duncan, Z. A. Sheikh, H.-J. Beyer, and H. Kleinpoppen, J. Phys. B 30, 1347 (1997).

[55] R. W. Dunford, E. P. Kanter, H. W. Schäffer, P. H. Mokler, H. G. Berry, A. E. Livingston, S. Cheng, and L. J. Curtis, Phys. Scr. T 80, 143 (1999).

[56] Y. B. Zel'dovich, Zh. Éksp. Teor. Fiz. 33, 1531 (1957), [JETP 6, 1184 (1958)].

[57] Y. B. Zel'dovich, Zh. Éksp. Teor. Fiz. 36, 964 (1959), [JETP 9, 682 (1958)].

[58] B. Sakitt and G. Feinberg, Phys. Rev. 151, 1341 (1966).

[59] R. Marrus and R. Schmieder, Phys. Rev. A 5, 1160 (1972). 